\title{
Concentration Fluctuations in Liquid Alloys of Alkali Metals from Semiempirical Theories of Mixtures
}

\author{
L. J. Gallego*, J. A. Alonso**, and J. A. Somoza* \\ Z. Naturforsch. 40 a, $425-429$ (1985); received December 1, 1984
}

\begin{abstract}
The semiempirical theories of mixtures developed by van Laar, Margules, Scatchard-Hamer, Wilson and Renon and Prausnitz are used to study the concentration fluctuations of binary liquid alloys formed among the alkali metals. Examination of the fitting capabilities of these theories leads to the conclusion that those of van Laar, Scatchard-Hamer and Renon and Prausnitz are appropriate both when the atomic sizes of the component metals are similar and when there is considerable atomic size mismatch. The Margules and Wilson theories, on the contrary, appear to be restricted only to cases in which the size difference between the two types of atoms is small.
\end{abstract}

\section{Introduction}

In recent years, a number of workers have discussed the characteristic features of the concentration fluctuation structure factor $S_{c c}(0)$ of a binary liquid alloy $A_{c} B_{1-c}$ using different models of mixtures [1-19]. In terms of the molar excess Gibbs energy, $g_{\mathrm{E}}$, the expression for $S_{c c}(0)$ is

$$
S_{c c}(0)=\frac{c(1-c)}{1+c(1-c)\left(\frac{\partial^{2}\left(g_{\mathrm{E}} / R T\right)}{\partial c^{2}}\right)_{T, P}},
$$

where $R$ is the gas constant. The simplest theories of mixtures are perhaps the lattice theories [20]. In principle, lattice models apply directly to solid mixtures, but in practice they are equally well applicable to liquids. Thus Bhatia [4] has used the theory of regular solutions (in the zeroth approximation [20]) to study the observed results for $S_{c c}(0)$ in liquid $\mathrm{Na}-\mathrm{K}$ alloys, and Bhatia and March [3] have shown that Flory's theory [21] (also a lattice model, but a more refined one) gives fairly good results for Na-Cs alloys. The regular and Flory models have recently been combined by Alonso et al. [11, 12] with Miedema's theory on heats of mixing [22] to study concentration fluctuations in simple liquid alloys. Regular and Flory models have also been used by Bhatia and Hargrove [23] to explain the experimental behaviour of $S_{c c}(0)$ in the so-called

\footnotetext{
* Departamento de Física Fundamental, Universidad de Santiago de Compostela, La Coruña, Spain.

** Departamento de Física Teórica, Universidad de Valladolid, Valladolid, Spain.

Reprint request to Dr. L. J. Gallego, Departamento de Física Fundamental, Universidad de Santiago de Compostela, La Coruña, Spain.
}

compound-forming alloys. More fundamental theories of mixtures, especially those based on a hard sphere model, have also been used successfully to analyse the concentration dependence of $S_{c c}(0)$ in various binary liquid alloys $[7,9,17]$.

Besides the aforementioned theories, there are many others which have been proposed for expressing analytically the dependence of $g_{\mathrm{E}}$ on composition. Most of them are semiempirical, suffering from the lack of a precise theoretical basis. However, they have been found to be very useful for interpreting and correlating thermodynamic data for a large variety of (mainly organic) mixtures [24]. In the present paper we shall consider two groups of these theories: on the one hand, the van Laar (VL), Margules (M) and Scatchard-Hamer (SH) theories, which have in common the possibility of deriving them from Wohl's general method for expressing the dependence of $g_{\mathrm{E}}$ on composition (see, e.g. Prausnitz's textbook [24]); and on the other hand, the Wilson (W) [25] and the non-random two liquid (NRTL) [26] theories, both based on the concept of local composition introduced originally by Wilson. Several of these theories have recently been used by Vrestal and Velisek [27] to model the composition dependence of $g_{\mathrm{E}}$ in different classes of solid and liquid metallic alloys. However, with the exception of the NRTL theory (which Gallego and Alonso [18] have recently fitted to the observed $S_{c c}(0)$ data for $\mathrm{Na}-\mathrm{Cs}$ liquid alloys), they do not appear to have been employed to study the behaviour of concentration fluctuations in liquid metallic alloys. In the present paper, we shall therefore compare the abilities of these models to fit the experimental $S_{c c}(0)$ data for various binary liquid alloys formed among the alkali metals. 


\section{Expressions for $S_{c c}(0)$ and Their Application to Liquid Alkali Alloys}

The expressions for the molar excess Gibbs energy of a binary mixture $A_{c} B_{1-c}$ in the VL, M, SH, W and NRTL models are respectively

$$
\begin{aligned}
\frac{g_{\mathrm{E}}^{\mathrm{VL}}}{R T}=c(1-c) \frac{u_{1} u_{2}}{u_{1} c+u_{2}(1-c)}, \\
\frac{g_{\mathrm{E}}^{\mathrm{M}}}{R T}=c(1-c)\left[u_{1}+u_{2}(2 c-1)\right], \\
\frac{g_{\mathrm{E}}^{\mathrm{SH}}}{R T}=c(1-c) \frac{u_{2} c+u_{1} r^{2}(1-c)}{[c+r(1-c)]^{2}}, \\
\frac{g_{\mathrm{E}}^{\mathrm{W}}}{R T}=-c \ln \left[c+\lambda_{1}(1-c)\right] \\
-(1-c) \ln \left[(1-c)+\lambda_{2} c\right],
\end{aligned}
$$

and

$$
\begin{aligned}
\frac{g_{\mathrm{E}}^{\mathrm{NRTL}}}{R T}= & c(1-c)\left[\frac{\tau_{1} e^{-\alpha \tau_{1}}}{c+(1-c) e^{-\alpha \tau_{1}}}\right. \\
& \left.+\frac{\tau_{2} e^{-\alpha \tau_{2}}}{(1-c)+c e^{-\alpha \tau_{2}}}\right] .
\end{aligned}
$$

In the above equations $u_{1}, u_{2}, \lambda_{1}, \lambda_{2}, \tau_{1}$ and $\tau_{2}$ are parameters to which some approximate physical significance can be assigned in terms of interactions between the components of the mixture [24-26]. In practice, however, they are considered as freely adjustable. In (4) $r$ is the volume ratio $V_{\mathrm{B}} / V_{\mathrm{A}}$, and $\alpha$ in (6) is a parameter characterizing the tendency of the components to mix in a nonrandom fashion. Differentiating (2) - (6) twice with respect to $c$ and using (1) yields the following expressions for $S_{c c}(0)$ :
In order to test the adequacy of (7) - (11), we shall consider the liquid alloys $\mathrm{K}-\mathrm{Cs}, \mathrm{Na}-\mathrm{K}$ and $\mathrm{Na}-\mathrm{Cs}$, whose behaviour is well documented. The data reported by Alblas et al. [28], Hultgren et al. [29] and Neale and Cusack [30] will be taken as the experimental references for the K-Cs, Na-K and NaCs systems, respectively. These liquid alloys between alkali metals represent a rather simple group, because they are all monovalent and have very similar electronegativities. However, there exist clear differences among them as regards the behaviour of $S_{c c}(0)$ (see below), which is correlated with the atomic volume ratios (approximately, $V_{\mathrm{Cs}} / V_{\mathrm{K}}=1.5, V_{\mathrm{K}} / V_{\mathrm{Na}}=2$ and $V_{\mathrm{Cs}} / V_{\mathrm{Na}}=3$ ).

The results of the calculations performed are given in Tables $1-3$ and Figures $1-3$. Tables $1-3$ contain the optimal values of the parameters of (7) - (11) for each system considered in this paper. These values were calculated using Marquardt's nonlinear optimum seeking procedure [31]. In the same tables we also give the standard deviations of the different fits as a measure of the success of each model. In the calculations we have always considered the two parameters of (7)-(10) as freely adjustable, as also the energy parameters $\tau_{1}$ and $\tau_{2}$ in the NRTL model. The non-randomness parameter $\alpha$ of this latter theory has only been considered as free for the system $\mathrm{Na}-\mathrm{Cs}$ in which the number of experimental data justify a three-parameter theory being considered. For the systems K-Cs and $\mathrm{Na}-\mathrm{K}$, for which there are fewer data points, we have fixed the value of $\alpha$ equal to 0.3 , which is in accord with the rule of thumb established by Renon

$$
\begin{aligned}
S_{c c}^{\mathrm{VL}}(0) & =\frac{c(1-c)}{1-\left[2 u_{1}^{2} u_{2}^{2} c(1-c) /\left(u_{1} c+u_{2}-u_{2} c\right)^{3}\right]}, \\
S_{c c}^{\mathrm{M}}(0) & =\frac{c(1-c)}{1-2 c(1-c)\left[u_{1}-3 u_{2}(1-2 c)\right]}, \\
S_{c c}^{\mathrm{SH}}(0) & =\frac{c(1-c)}{1+c(1-c) \frac{2 u_{2} r^{2}(1-c)-4 u_{2} r c-4 u_{1} r^{3}(1-c)+2 u_{1} r^{2} c}{[c+r(1-c)]^{4}},} \\
S_{c c}^{\mathrm{W}}(0) & \left.=\frac{c(1-c)}{1+c(1-c)\left[\frac{\lambda_{2}\left(\lambda_{2}-1\right)}{\left(c+\lambda_{1}(1-c)\right)\left((1-c)+\lambda_{2} c\right)}+\frac{\lambda_{1} \lambda_{2}-1}{\left((1-c)+\lambda_{2} c\right)^{2}}\right.}+\frac{\lambda_{1}\left(\lambda_{1}-1\right)}{\left(c+\lambda_{1}(1-c)\right)^{2}}\right],
\end{aligned}
$$

and

$$
S_{c c}^{\mathrm{NRTL}}(0)=\frac{c(1-c)}{1-2 c(1-c)\left[\frac{\tau_{1} e^{-2 \alpha \tau_{1}}}{\left(c+(1-c) e^{-\alpha \tau_{1}}\right)^{3}}+\frac{\tau_{2} e^{-2 \alpha \tau_{2}}}{\left((1-c)+c e^{-\alpha \tau_{2}}\right)^{3}}\right]} .
$$


Table 1. Optimal values of the parameters and standard deviations of the fits of the various models considered in this paper to the experimental $S_{c c}(0)$ data for the K-Cs system.

\begin{tabular}{llll}
\hline Theory & Values of the parameters & $\begin{array}{l}\text { Standard } \\
\text { deviation }\end{array}$ \\
\hline VL & $u_{1}=0.094$ & $u_{2}=0.44$ & 0.018 \\
M & $u_{1}=0.128$ & $u_{2}=0.117$ & 0.018 \\
SH & $u_{1}=0.086$ & $u_{2}=0.29$ & 0.018 \\
W & $\lambda_{1}=1.81$ & $\lambda_{2}=0.32$ & 0.018 \\
NRTL & $\tau_{1}=-0.84 \quad \tau_{2}=1.35$ & 0.018 \\
& \multicolumn{2}{c}{$\alpha=0.3$}
\end{tabular}

Table 2. Optimal values of the parameters and standard deviations of the fits of the various models considered in this paper to the experimental $S_{c c}(0)$ data for the $\mathrm{Na}-\mathrm{K}$ system.

\begin{tabular}{llll}
\hline Theory & \multicolumn{2}{c}{ Values of the parameters } & $\begin{array}{l}\text { Standard } \\
\text { deviation }\end{array}$ \\
\hline VL & $u_{1}=0.893$ & $u_{2}=0.992$ & 0.012 \\
M & $u_{1}=0.938$ & $u_{2}=0.053$ & 0.011 \\
SH & $u_{1}=0.8106$ & $u_{2}=0.800$ & 0.0094 \\
W & $\lambda_{1}=0.644$ & $\lambda_{2}=0.510$ & 0.014 \\
NRTL & $\tau_{1}=0.334$ & $\tau_{2}=0.698$ & 0.012 \\
& \multicolumn{2}{c}{$\alpha=0.3$} \\
\hline
\end{tabular}

Table 3. Optimal values of the parameters and standard deviations of the fits of the various models considered in this paper to the experimental $S_{c c}(0)$ data for the $\mathrm{Na}-\mathrm{Cs}$ system.

\begin{tabular}{llll}
\hline Theory & Values of the parameters & $\begin{array}{l}\text { Standard } \\
\text { deviation }\end{array}$ \\
\hline VL & $u_{1}=0.872$ & $u_{2}=2.456$ & 0.055 \\
M & $u_{1}=0.48$ & $u_{2}=1.214$ & 0.14 \\
SH & $u_{1}=0.870$ & $u_{2}=2.453$ & 0.055 \\
W & $\lambda_{1}=0.743 \quad \lambda_{2}=0.0432$ & 0.13 \\
NRTL & $\tau_{1}=-0.18 \quad \tau_{2}=2.60$ & 0.055 \\
& \multicolumn{2}{r}{$\alpha=0.37$} \\
\end{tabular}

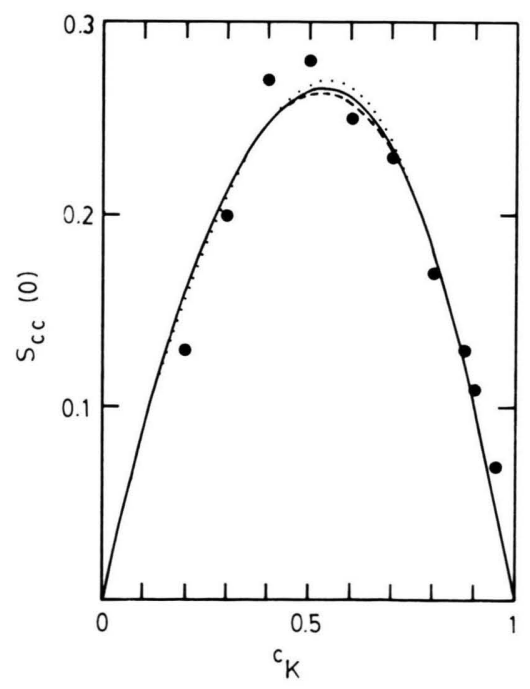

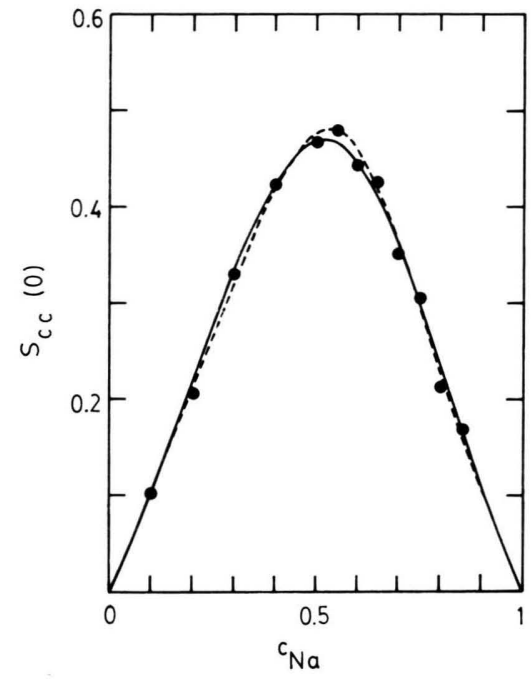

Fig. 2. $S_{c c}(0)$ of the liquid alloy $\mathrm{Na}-\mathrm{K}$ as a function of $\mathrm{Na}$ concentration. ( $\bullet$ Experimental data at $T=111^{\circ} \mathrm{C}$, after Hultgren et al. [29]. (-) VL, M, W and NRTL models; (---) SH model.

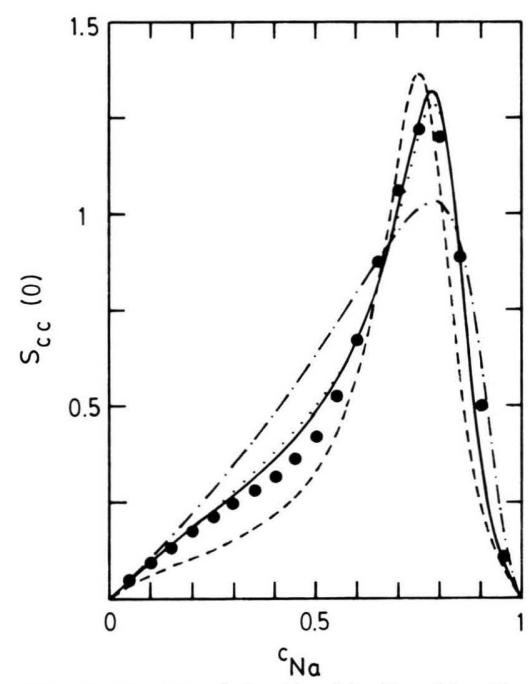

Fig. 3. $S_{c c}(0)$ of the liquid alloy $\mathrm{Na}-\mathrm{Cs}$ as a function of $\mathrm{Na}$ concentration. ( $\bullet$ Experimental data at $T=110^{\circ} \mathrm{C}$, after Neale and Cusack [30]; (---) M model; ( -$)$ NRTL model; $(\cdot \cdot)$ VL and SH models; $(\cdot-\cdot-\cdot)$ W model.

Fig. 1. $S_{c c}(0)$ of the liquid alloy K-Cs as a function of $\mathrm{K}$ concentration. $(\bullet)$ Experimental data at $T=100^{\circ} \mathrm{C}$, after Alblas et al. [28]; (---) VL model; (-) W and NRTL models; $(\cdots)$ M model; the curve corresponding to the $\mathrm{SH}$ model is between that of the $\mathrm{W}$ and NRTL models and that of the $\mathrm{M}$ model in the range from $c_{\mathrm{K}}=0.4$ to $c_{\mathrm{K}}=0.7$. 
and Prausnitz [26] for systems with no large deviations from ideal behaviour.

By comparing the results of the fits for the various models considered in this paper, several conclusions can be drawn. For the K-Cs system all the models are equally successful, as can be seen in Table 1 and Figure 1. This is a consequence of the very simple nature of the K-Cs liquid alloy, which is in fact a nearly-ideal solution, as has been pointed out by Hafner [32] and by Alblas [33]. For the Na-K system, a borderline case for treatment as a regular solution because the volume ratio $V_{\mathrm{K}} / V_{\mathrm{Na}}$ is about 2 $[4,20]$, all models can also be considered as suitable. There exist however small differences in favour of the SH model for this system, especially in the region around $c_{\mathrm{Na}}=0.5$, as can be appreciated in Figure 2. The differences between the various models are more significant for the system $\mathrm{Na}-\mathrm{Cs}$ (see Table 3 and Figure 3). In this case, the VL and $\mathrm{SH}$ models provide much better results than the $\mathrm{M}$ model. This is consistent with Wohl's method for deriving these three models, because the latter corresponds precisely to the assumption that the size difference is not important [24]. This explains why the $\mathrm{M}$ model does a good job for $\mathrm{K}-\mathrm{Cs}$ and for $\mathrm{Na}$ $\mathrm{K}$, whereas the results for $\mathrm{Na}-\mathrm{Cs}$ are worse. It may also be noted that of the two theories based on the concept of local composition ((10) and (11)) the best fit for Na-Cs is provided by the NRTL model, the results being comparable with those obtained with the VL and SH models. With regard to the NRTL theory, a few remarks about the physical meaning of the energy parameters $\tau_{1}$ and $\tau_{2}$ may be in order. These are defined by $\tau_{1}=\left(g_{\mathrm{AB}}-g_{\mathrm{AA}}\right) / R T$ and $\tau_{2}=\left(g_{\mathrm{AB}}-g_{\mathrm{BB}}\right) / R T$, where the $g$ 's are (Gibbs) energy parameters characterizing the interaction between the components denoted by the subscripts. The quantity $w \equiv\left(\tau_{1}+\tau_{2}\right) / 2=\left(2 g_{\mathrm{AB}}-g_{\mathrm{AA}}-g_{\mathrm{BB}}\right) / 2 R T$ can thus be considered as a parameter determining the character of the mixture: $w>0$ indicates clustering or phase separation and $w<0$ compound formation. For the systems considered in this paper the values of $w$ are $w(\mathrm{~K}-\mathrm{Cs})=0.255, w(\mathrm{Na}-\mathrm{K})$ $=0.516$ and $w(\mathrm{Na}-\mathrm{Cs})=1.21$, which shows that in this order, these systems exhibit an increasing tendency for phases to separate out.

To sum up, the VL, SH and NRTL theories can be helpful in studies of the concentration fluctuations of liquid alloys both in cases in which the atomic sizes of the component metals are similar and when the atomic size mismatch is relatively large. This has been tested in this paper by considering binary liquid alloys formed among the alkali metals. The $\mathrm{M}$ and $\mathrm{W}$ theories, however (the other two models here considered), appear to be restricted to mixtures in which the size difference between the two types of atoms is small.

\section{Acknowledgements}

This work was supported in part by a Grant from the Spanish Comisión Asesora de Investigación Científica y Técnica.
[1] A. B. Bhatia and D. E. Thornton, Phys. Rev. B 2, 3004 (1970).

[2] A. B. Bhatia, W. H. Hargrove, and N. H. March, J. Phys. C: Solid State Phys. 6, 621 (1973).

[3] A. B. Bhatia and N. H. March, J. Phys. F: Metal Phys. 5, 1100 (1975).

[4] A. B. Bhatia, Liquid Metals, Inst. Phys. Conf. Ser. No. 30, p. 21 (1977).

[5] N. H. March and M. P. Tosi, Atomic Dynamics in Liquids, MacMillan, London 1976.

[6] J. C. Thompson, K. Ichikawa, and S. M. Granstaff, Phys. Chem. Liquids 5, 167 (1976).

[7] V. K. Ratti and A. B. Bhatia, Il Nuovo Cim. 43 B, 1 (1978).

[8] P. Gray, N. E. Cusack, S. Tamaki, and Y. Tsuchiya, Phys. Chem. Liquids 9, 307 (1980).

[9] E. G. Visser, W. van der Lugt, and J. Th. M. de Hosson, J. Phys. F: Metal Phys. 10, 1681 (1980).

[10] B. P. Alblas, W. van der Lugt, E. G. Visser, and J. Th. M. de Hosson, Physica 114 B, 59 (1982).
[11] J. A. Alonso and N. H. March, Physica 114 B, 67 (1982).

[12] J. A. Alonso, J. M. López, and N. H. March, J. de Physique-Lettres 43, L 441 (1982).

[13] S. Tamaki, Y. Waseda, S. Takeda, and Y. Tsuchiya, J. Phys. F: Metal Phys. 12, 1101 (1982).

[14] A. B. Bhatia and R. N. Singh, Phys. Chem. Liquids 11, 285, 343 (1982)

[15] N. Ohtomo and K. Arakawa, J. Phys. Soc. Japan 51, $1282(1982)$

[16] Y. Tanaka, N. Ohtomo, and K. Arakawa, J. Phys. Soc. Japan 52, 2093 (1983)

[17] F. E. Neale and N. E. Cusack, J. Non-Crystalline Solids 61 and 62, 169 (1984).

[18] L. J. Gallego and J. A. Alonso, Z. Naturforsch. 39a, 596 (1984).

[19] R. N. Singh and A. B. Bhatia, J. Phys. F: Metal Phys. 14, 2309 (1984)

[20] A. E. Guggenheim, Mixtures, Oxford University Press, Oxford 1952 
[21] P. J. Flory, J. Chem. Phys. 10, 51 (1942).

[22] A. R. Miedema, P. F. de Châtel, and F. R. de Boer, Physica 100 B, 1 (1980).

[23] A. B. Bhatia and W. H. Hargrove, Phys. Rev. B 10, 3186 (1974).

[24] J. M. Prausnitz, Molecular Thermodynamics of Fluid Phase Equilibria, Prentice-Hall, New York 1969.

[25] G. M. Wilson, J. Amer. Chem. Soc. 86, 127 (1964).

[26] H. Renon and J. M. Prausnitz, A. I. Chem. Eng. J. 14, 135 (1968).

[27] J. Vrestal and J. Velisek, CALPHAD 6, 297 (1982).

[28] B. P. Alblas, W. van der Lugt, O. Mensies, and C. van Dijk, Physica 106 B, 22 (1981).
[29] R. R. Hultgren, P. D. Desai, D. T. Hawkins, M. Gleiser, and K. K. Kelley, Selected Values of the Thermodynamic Properties of Binary Alloys, Amer. Soc. Metals, Ohio 1973.

[30] F. E. Neale and N. E. Cusack, J. Phys. F: Metal Phys. 12, 2839 (1982)

[31] D. W. Marquardt, J. Soc. Indust. Appl. Math. 11, 431 (1963).

[32] J. Hafner, Phys. Rev. B 15, 617 (1977).

[33] B. P. Alblas, Thesis, University of Groningen, The Netherlands (1983). 